\title{
Response to letter to the editor; TEVAR for a Marfan patient during COVID-19 pandemic.
}

\author{
Shinichiro Ikeda ${ }^{1}$, Michael Shih ${ }^{1}$, Robert Rhee ${ }^{1}$, and Benjamin Youdelman ${ }^{1}$ \\ ${ }^{1}$ Maimonides Medical Center
}

July 7,2020

Dear Dr Harky et. al,

We appreciate your inquiry regarding our case report. Dr Harky et. al suggested that TEVAR for a Marfan patient could be an unnecessary approach even during the COVID-19 pandemic.

We believe in this particular case, the endovascular approach was fully justified as the patient had clear signs of end organ ischemia at presentation. He presented with extreme right leg ischemia with diffuse numbness. There was no detectable distal arterial flow of the right extremity by a Doppler and physical evaluation. Contrast computed tomography scan showed a completely occluded right common iliac artery and diminished flow to the right renal and celiac arteries due to the compression of the true lumen from the false lumen. Preoperative creatinine was elevated to $1.2 \mathrm{mg} / \mathrm{dl}$. She was also suffering ongoing right kidney malperfusion.

It was during the time when COVID-19 epidemic started spreading rapidly in New York City. Our hospital beds were filled with COVID-19 patients and there was a shortage of medical supplies with no ventilators immediately available. It was important to reduce exposure of the individual to the hospital environment and minimize length of stay and ventilator needs. As such, we chose to proceed with TEVAR to minimize the risk of lung injury which can occur in open repair. Postoperative respiratory failure is a major issue in open thoracic aortic repair [1]. The patient did not have a risk of respiratory comorbidities but we believed that this pandemic placed all patients at risk for contracting COVID-19 and subsequent acute respiratory distress [2].

Due to the high risk of spinal cord ischemia in this particular patient, we performed TEVAR with a distal bare metal component to preserve the blood flow into spinal cord arteries [3]. The initial clinical treatment plan was to perform the TEVAR as a bridge to open repair. We obviously will need to follow-up with her carefully and if any signs of failure of TEVAR is detected, open repair will ultimately be required.

Dr Harky et. al suggested axillary femoral artery bypass to rescue the ischemic leg, however, this patient also suffered malperfsuion of the renal and celiac arteries, so further intervention was required.

Thank you for your insightful suggestions.

References

1) Khan FM, Naik A, Hameed I, et al. Open repair of descending thoracic and thoracoabdominal aortic aneurysms: a meta-analysis. Ann Thorac Surg . 2020;S0003-4975(20)30865-1.

2) Bai Y, Yao L, Wei T, et al. Presumed Asymptomatic Carrier Transmission of COVID-19. JAMA. 2020;323:1406-7.

3) Lombardi JV, Cambria RP, Nienaber CA, et al. Five-year results from the study of Thoracic Aortic Type B Dissection Using Endoluminal Repair (STABLE I) study of endovascular treatment of complicated type 
B aortic dissection using a composite device design. J Vasc Surg. 2019; 70:1072-81. 